\title{
Alterstice
}

Revue internationale de la recherche interculturelle

International Journal of Intercultural Research

Revista International de la Investigacion Intercultural

\section{Plurilinguisme à géométrie variable des commerçants sur le marché Saint-Maur de Ziguinchor : usages fonctionnels des langues dans les échanges commerciaux}

\section{Jean Sibadioumeg Diatta}

Volume 7, numéro 2, 2017

Diversité(s) au coeur des politiques et des pratiques : entre l'Europe et l'Afrique

URI : https://id.erudit.org/iderudit/1052567ar

DOI : https://doi.org/10.7202/1052567ar

Aller au sommaire du numéro

Éditeur(s)

Alterstice

ISSN

1923-919X (numérique)

Découvrir la revue

Citer cet article

Diatta, J. S. (2017). Plurilinguisme à géométrie variable des commerçants sur le marché Saint-Maur de Ziguinchor : usages fonctionnels des langues dans les échanges commerciaux. Alterstice, 7(2), 23-36.

https://doi.org/10.7202/1052567ar

\section{Résumé de l'article}

À Ziguinchor, l'un des éléments qui frappe tout visiteur reste la cohabitation de locuteurs de langues différentes. Que ce soient dans les familles, les écoles, les marchés ou même les rues, plusieurs langues sont quotidiennement utilisées dans les discours et les échanges. Le marché Saint-Maur, le principal de la ville, est marqué par la pluralité linguistique. Cependant, il faut noter qu'avec la montée en puissance du wolof dans la communication commerciale, nous assistons à l'apparition de phénomènes liés notamment à la distribution fonctionnelle des idiomes. D’un plurilinguisme pratiqué par une grande majorité de Sénégalais, tant chanté par Juillard dans le passé, nous arrivons à un plurilinguisme diversifié et moins homogène. Ainsi, les pratiques linguistiques dans les cantines de vente d'habits et autres produits manufacturés sont bien différentes de celles que l'on retrouve dans les étals. Nous avons identifié sur le marché Saint-Maur un plurilinguisme à géométrie variable. Pour faire face à la diversité, les vendeurs développent des stratégies d'adaptation à la langue de leurs acheteurs en cherchant à maîtriser des connaissances linguistiques minimales mais suffisantes pour pouvoir échanger et marchander. 


\section{7}

ARTICLE THÉMATIQUE

\section{Plurilinguisme à géométrie variable des commerçants sur le marché Saint-Maur de Ziguinchor : usages fonctionnels des langues dans les échanges commerciaux}

Jean Sibadioumeg Diatta ${ }^{1}$

\section{Résumé}

À Ziguinchor, l'un des éléments qui frappe tout visiteur reste la cohabitation de locuteurs de langues différentes. Que ce soient dans les familles, les écoles, les marchés ou même les rues, plusieurs langues sont quotidiennement utilisées dans les discours et les échanges. Le marché Saint-Maur, le principal de la ville, est marqué par la pluralité linguistique. Cependant, il faut noter qu'avec la montée en puissance du wolof dans la communication commerciale, nous assistons à l'apparition de phénomènes liés notamment à la distribution fonctionnelle des idiomes. D’un plurilinguisme pratiqué par une grande majorité de Sénégalais, tant chanté par Juillard dans le passé, nous arrivons à un plurilinguisme diversifié et moins homogène. Ainsi, les pratiques linguistiques dans les cantines de vente d'habits et autres produits manufacturés sont bien différentes de celles que l'on retrouve dans les étals. Nous avons identifié sur le marché Saint-Maur un plurilinguisme à géométrie variable. Pour faire face à la diversité, les vendeurs développent des stratégies d'adaptation à la langue de leurs acheteurs en cherchant à maîtriser des connaissances linguistiques minimales mais suffisantes pour pouvoir échanger et marchander.

\section{Rattachement de l'auteur \\ ${ }^{1}$ Université Cheikh Anta Diop, Dakar, Sénégal}

\section{Correspondance}

jean-sibadioumeg.diatta@ugb.edu.sn

\section{Mots clés}

sociolinguistique urbaine, plurilinguisme, wolof, usages fonctionnels des langues, pratiques linguistiques « à géométrie variable », Sénégal, Ziguinchor

\section{Pour citer cet article}

Diatta, J. S. (2017). Plurilinguisme à géométrie variable des commerçants sur le marché Saint-Maur de Ziguinchor : usages fonctionnels des langues dans les échanges commerciaux. Alterstice, 7(2), 23-36. 


\section{Introduction}

Le continent africain compte plus de 800 langues premières autochtones, regroupées en quatre familles. Ces langues et leurs dialectes sont en contact entre elles ainsi qu'avec les langues ex-coloniales, l'anglais, le français et le portugais, etc. Cette situation pose la question de la diversité linguistique en Afrique (Calvet, 1991, p. 418). La cohabitation de plusieurs ethnies et de langues dans un espace commun est l'une des particularités des milieux urbains. Point de convergence de populations issues de divers horizons, la ville constitue le lieu par excellence de la rencontre, mais aussi de la coexistence des langues et des cultures. C'est dans cette situation de multilinguisme que s'inscrit la ville de Ziguinchor qui reste, de l'avis de Calvet, " un bel exemple de ce type de situation » (cité dans Juillard, 1995, p. 12). On y utilise une vingtaine de langues locales auxquelles se superposent la langue officielle, le français, et la langue véhiculaire venue du Nord, le wolof. Les marchés constituent des espaces privilégiés des sociolinguistes pour étudier la diversité des pratiques linguistiques dans les échanges professionnels. Le plurilinguisme est au cœur des activités commerciales, car chaque acteur y vient avec sa ou ses langue(s). Savoir manier plusieurs langues, notamment dans des échanges commerciaux, traduit bien l'opportunité de développer ses activités car, selon Bourdieu (1982, p. 13) le langage est un instrument à la fois " d'action et de pouvoir ».

Par ailleurs, des études antérieures sur Ziguinchor ont montré que le plurilinguisme des locuteurs de la ville de Ziguinchor est une pratique normale au quotidien. Ils sont généralement capables de passer d'une langue à l'autre selon les situations discursives. II s'agit de ce que Juillard (1995, p. 19) considère comme " un marqueur identitaire de cette population citadine ". Cependant, il faut noter qu'avec la montée en puissance du wolof dans la communication commerciale, nous assistons à l'émergence de phénomènes liés notamment à la distribution fonctionnelle des idiomes. Du plurilinguisme tant changé par Juillard, nous arrivons à un plurilinguisme diversifié et moins homogène.

Dans ce texte, l'analyse des interviews réalisées et des interactions recueillies sur le marché Saint-Maur permettra de mettre en relief les nouvelles dynamiques plurilingues plus d'une vingtaine d'années après les études de Juillard. Il s'agit aussi de voir comment les acteurs s'adaptent aux rapports entre les langues en développant des stratégies pour faciliter la communication et faire aboutir les échanges commerciaux.

Nous proposons trois étapes de réflexion dans cet article. Dans un premier temps, nous brosserons un tableau de la sociolinguistique urbaine dans laquelle s'inscrit ce travail, avec un accent particulier sur le contexte de Ziguinchor. Dans un deuxième temps, nous passerons en revue le contexte, le terrain d'étude ainsi que la méthodologie adoptée par recueillir les données. Enfin, nous analyserons les nouvelles dynamiques du multilinguisme urbain.

\section{Bref aperçu sur les recherches en sociolinguistique urbaine}

Selon la littérature, c'est précisément au début de la seconde moitié du $20^{\mathrm{e}}$ siècle, notamment avec la parution des premiers travaux de Labov sur Martha's Vineyard, que la sociolinguistique a émergé comme discipline à part entière (Dumont et Maurer, 1995, p. 135). Situé au carrefour de la linguistique et de la sociologie, et bénéficiant également d'apports d'autres disciplines, la sociolinguistique est désormais comprise comme l'analyse processuelle des rapports entre langues, locuteurs et sociétés. Elle constitue une discipline prenant en compte tous les phénomènes liés à l'homme parlant au sein d'une société. La sociolinguistique considère donc la langue comme une production, un acte social (Calvet, 1994, p. 77).

De la sociolinguistique jaillit la sociolinguistique urbaine, dont la définition divise les spécialistes. Bulot (2009) la rapproche de la " géographie sociale " car il considère que l'espace n'est pas une donnée, mais un produit, une construction sociale, que l'action humaine est une dimension spatiale, que d'une part « la géographie sociale est sensible à la désignation, à la mise à mots de l'espace (Veschambre, 2004) et que d'autre part « les discours sur la ville modifient la perception du réel urbain » ou que " les discours sur la ville finissent par devenir la ville » (Bulot et Veschambre, 2004). Veschambre (2004, p. 1) distingue une sociolinguistique " classique ", qui étudie la covariance langue/société, sans problématiser la ville et la " sociolinguistique urbaine » qui considère que l'espace est un produit social, que la dénomination et la désignation de l'espace concourent à le produire socialement. Cependant, au-delà de la pluralité conceptuelle, nous adoptons la vision de Calvet qui la conçoit comme " une analyse de la 
mise en mots de la ville " (2005, p. 16), car la ville est le lieu par excellence de contacts de langues résultant de I'urbanisation et de la migration. Calvet (p. 11) distingue trois orientations de la sociolinguistique urbaine. II note d'abord l'analyse des rapports entre les langues dans les villes plurilingues. II s'agit donc de la gestion in vivo du plurilinguisme. C'est ce volet qui fait l'objet de notre présente étude. Le deuxième axe s'intéresse à la ville définie " par sa mise en mots, par l'appropriation des lieux à travers la langue, avec un accent mis sur l'analyse du discours et plus récemment une approche interdisciplinaire, en particulier en relation avec la géographie sociale » (p. 11.). Le troisième courant concerne la ville considérée comme productrice lexicale avec notamment des études portant sur le langage des jeunes dans les cités, les banlieues, etc.

\section{Contexte, terrain d'études et méthodologie}

\section{Ziguinchor : une ville au carrefour de peuples et de langues}

Ziguinchor constitue un ancien comptoir commercial établi vers la fin du $17^{\mathrm{e}}$ siècle par les Portugais, et situé au bord du fleuve Casamance à proximité de l'ancienne route Nord Sud, de Gambie à Cacheu, colonie portugaise (Guinée-Bissau). Longtemps peuplé par les Créoles et les Baïnouks, premiers occupants du lieu, le comptoir s'est agrandi lorsque, dans le sillage des Français, qui ont pris la place des Portugais, une population migrante est venue s'établir par étapes, du reste du Sénégal ou des pays avoisinants (Dreyfus et Juillard, 2005, p. 42).

Au cours de son histoire, Ziguinchor a été un véritable carrefour humain, drainant des populations originaires non seulement du Sénégal, de l'Afrique occidentale, mais aussi de l'Europe. Ces groupes d'individus dont les raisons principales de l'immigration sont pour la plupart économiques avec le développement du commerce, politiques avec la mise en place de l'administration sénégalaise, mais aussi sociales avec l'exode rural, ont joué des rôles déterminants dans la construction culturelle et linguistique de la ville (Trincaz, 1984, p. 54). Certaines langues parlées à Ziguinchor appartiennent à la famille Niger-Congo. Le groupe ouest-atlantique nord regroupe les langues dites " sénégalaises " (le wolof, le sérère, le pulaar), les langues bak (le joola, le balant) ainsi qu'une langue du Sénégal oriental, le baïnouk. Le groupe mandé est constitué du mandinka, soninké, sarakholé et malinké.

Deux facteurs sont considérés comme ayant contribué à l'expansion du wolof en Casamance, particulièrement à Ziguinchor. Il s'agit en premier lieu du développement du commerce. En effet, les richesses naturelles casamançaises ont favorisé des déplacements de populations du reste du Sénégal pour les besoins du commerce. On note la présence massive des Wolof dans les marchés. En second lieu, leur arrivée résulte de la mise en place de l'administration au lendemain des indépendances de 1960 : plus de $80 \%$ des administratifs étaient originaires du Nord (Juillard, 1991, p. 440). Dès lors, pour discuter avec les autorités, le français et le wolof étaient les deux langues à utiliser, le français seul ne suffisant pas. Cette exigence de parler wolof fait penser au pouvoir du langage tel que le conçoit Bourdieu (1982, p. 21) quand il déclare : "la parole fait advenir. Le simple fait de dire peut également faire être, faire exister ».

\section{Méthodologie}

Notre enquête dans le marché Saint-Maur de Ziguinchor a été essentiellement ethnographique. II s'agit de procéder à une démarche interactionniste pour recueillir ce que Coulon (2014) appelle " les faits sociaux ». Nous avons utilisé d'une part les observations et d'autre part les entretiens semi-directifs à l'aide d'une grille de questions. Pour les observations des pratiques des acteurs, nous avons pu recueillir à l'aide de notre dictaphone 45 interactions. Issue de l'ethnologie, l'observation participante constitue la principale méthode de collecte de données dans une perspective d'étude qualitative en sciences sociales (Atsa Ncho, 2011). Quant aux entretiens semi-directifs, notre population cible est constituée de commerçants choisis au hasard et issus de tous les secteurs commerciaux. Au total, 35 entrevues comprises entre six et dix minutes ont été recueillies en wolof ou en diola, les langues locales que nous maitrisons. Le choix de ces deux méthodes de collectes se justifie par leur complémentarité, car les entrevues permettent de tester et de prouver les faits révélés par les observations. 


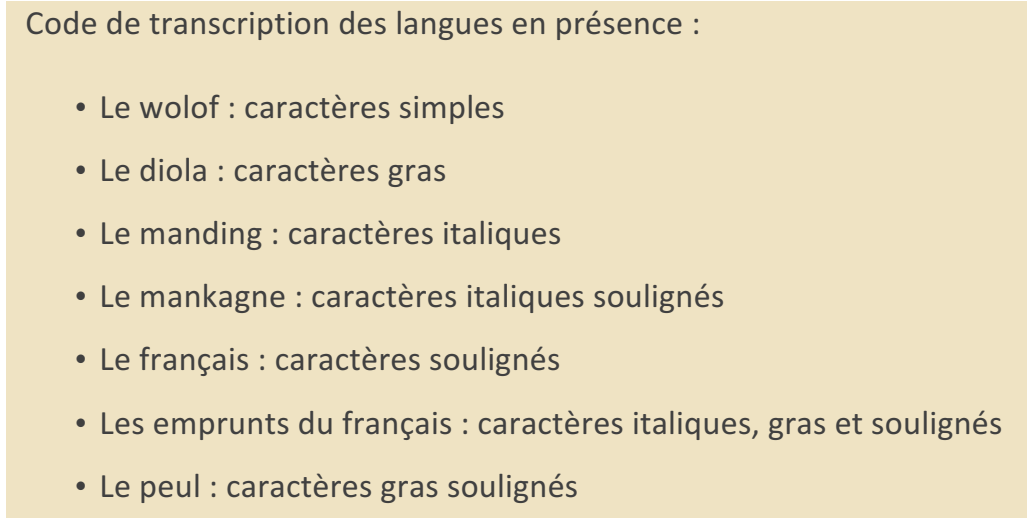

\section{Analyses : quelles dynamiques plurilingues actuelles à Saint-Maur?}

La présence de plusieurs langues dans un espace favorise l'émergence d'une ou de plusieurs langues véhiculaires. Au marché Saint-Maur, le wolof continue de jouer pleinement ce rôle de langue privilégiée et dominante, tout comme dans les autres secteurs de la vie sociale urbaine.

\section{Analyse des interactions commerciales}

Plusieurs schémas interactionnels peuvent se présenter dans l'interprétation du corpus. Cependant, en fonction de nos objectifs de recherche, nous nous appuierons sur les trois suivants: l'équilibre linguistique, l'adaptation du client à la langue du vendeur et l'adaptation du vendeur à la langue du client.

\section{La situation d'équilibre linguistique}

En analysant cette discussion entre deux Diola, nous remarquons l'usage de trois langues : outre le wolof, le diola et le français.

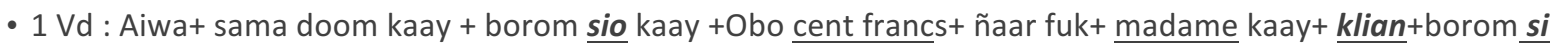

- $2 \mathrm{Cl}$ : Saam ñaata?

- $3 \mathrm{Vd}$ : Saam yii deux cents+ yii ñaar fuk

- $4 \mathrm{Vd}$ : Oumou wa du jaalo+ promonade?

\section{- $5 \mathrm{Cl}$ : Eeh promonade+ jat lecole bajut}

Traduction :

- $1 \mathrm{Vd}$ : Je vends+ ma fille viens + propriétaire de seau viens + sardinelle à cent francs + cent francs + madame viens + client + propriétaire de seau viens

- $2 \mathrm{Cl}$ : Le tas à combien?

- $3 \mathrm{Vd}$ : Celui-ci à deux cents + et l'autre à cent francs

- $4 \mathrm{Vd}$ : Oumou qu'est-ce que tu viens faire ici + promenade?

- $5 \mathrm{Cl}$ : Oui je viens me promener car il n'y a pas école aujourd'hui.

Le français se présente encore avec les expressions servant à indiquer le prix, mais aussi sous la forme d'emprunts dont les mots connaissent des modifications phonologiques. C'est le cas, par exemple, du wolof «sio » qui signifie " seau " en français, mais aussi de "klian » qui veut dire "client ». La vendeuse débute son intervention en wolof par diverses formules d'appels en direction de sa cliente. Pour sa part, celle-ci préfère la suivre en se servant du 
wolof pour demander le prix de la marchandise. Dans sa réponse, la vendeuse fait usage de français " $\underline{\text { deux cents" }}$ et du wolof «ñaar fuk » pour exprimer les prix de la marchandise. L'interprétation que nous pouvons donner à cette alternance est qu'elle est surtout motivée par le souci de compréhension de la cliente. En effet, la plupart des locuteurs non wolofs sont plus à l'aise avec l'usage du français pour exprimer les sommes d'argent. Par contre, certains montants standards comme "ñaar fuk », "cent francs » peuvent être alternés sans difficulté de compréhension de la part des locuteurs.

La suite de l'interaction est faite en diola. En abordant la jeune fille, la vendeuse ne connaissait pas son identité. C'est la raison pour laquelle elle lui parle en utilisant le wolof, mais aussi en la nommant " ma fille ». Par la suite, dès qu'elle l'a reconnue, elle change de langue en recourant à celle qui leur est commune, le diola. Ce que nous pouvons comprendre par là c'est que, comme nous l'avons affirmé ci-dessus, au marché le wolof sert à aborder une cliente et à décliner son identité.

Par ailleurs, après avoir reconnu la fille, la vendeuse comprend qu'elle n'est pas venue pour faire des achats, c'est la raison pour laquelle elle lui demande si elle est venue se promener. En fait, la jeune fille est une élève qui vient régulièrement aider sa maman vendeuse quand elle ne va pas à l'école. L'usage du terme "promonade" (promenade), qui est un emprunt lexical au français ayant subi de légères modifications, permet à la fille de justifier l'objet de sa présence au marché (elle vient au marché non pas pour faire des achats, mais pour se promener car elle n'a pas cours). À travers le terme "lecole » qui est aussi un emprunt intégré par le diola illustre la place importante de l'école à Ziguinchor. En effet, le rapport de l'ANSD (2013, p. 81) rapporte que Ziguinchor, malgré sa situation géographique et la crise armée, est la région la mieux alphabétisée au Sénégal en ce qui concerne la population de 10 ans et plus, à 62,3\%, contre 61,9\% à Dakar.

\section{Le client s'adapte au vendeur}

Dans les interactions à caractère commercial marquées par une situation de plurilinguisme, le vendeur, même si son intention est d'écouler rapidement ses produits en séduisant ses clients, impose quelquefois sa langue. Ainsi, ces deux discussions illustrent à bien des égards ce fait.

- $1 \mathrm{Cl}$ : Mbus ñaata?

- $2 \mathrm{Vd}$ : Been cent francs!

- $3 \mathrm{Cl}$ : Doo wañ̃̃i ?

- $4 \mathrm{Vd}$ : E jertai e se seer+moo kaane

- $5 \mathrm{Cl}$ : U bonketi waay!

Traduction :

- $1 \mathrm{Cl}$ : Le sachet (pâte d'arachide) coûte combien?

- 2 Vd : L'unité à cent francs

- $3 \mathrm{Cl}$ : Tu ne peux pas diminuer?

- $4 \mathrm{Vd}$ : C'est parce que l'arachide est chère.

- $5 \mathrm{Cl}$ : Aie pitié de lui vraiment!

II s'agit d'une interaction entre une cliente mankagne et une vendeuse diola. La discussion commence par l'abord de la cliente en wolof pour demander le prix du sachet de la pâte d'arachide. Elle se poursuit dans cette langue jusqu'au moment où la cliente souhaite la réduction du prix. La suite de l'interaction se fait en diola. En effet, sachant le besoin pressant de la cliente d'acheter la marchandise, la vendeuse répond en diola, une façon pour elle de lui imposer sa langue. La position de force détermine donc le choix de l'alternance dans les échanges. N'ayant probablement pas de recours, la cliente a été obligée de se débrouiller pour la suivre en s'exprimant dans l'idiome de la vendeuse avec certaines incorrections. En effet, au lieu de dire « u bonketom » (trad : " aie pitié de moi ») elle 
déclare « u bonketi » (trad : " aie pitié de toi ». Cette incompétence se traduit également par le mélange qu’elle fait du diola avec le wolof à travers « U bonketi waay! ", c'est-à-dire " Aie pitié de lui vraiment! ".

La seconde interaction traduisant l'adaptation de la cliente au choix linguistique de la vendeuse concerne une femme manding venue faire ses achats et qui demande à une commerçante diola de lui faire la monnaie. Voici l'extrait :

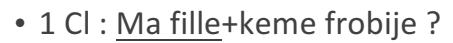

- 2 Vd : Deedet!

- $3 \mathrm{Cl}$ : Patron+amulen cinq cents wekket

- $4 \mathrm{Vd}$ : Mani la deedet +xana yow doo degg ?

Traduction :

- $1 \mathrm{Cl}$ : Ma fille + tu n'as pas une monnaie de cinq cents?

- $2 \mathrm{Vd}$ : Non!

- $3 \mathrm{Cl}$ : Patron + tu n'as pas la monnaie de cinq cents?

- $4 \mathrm{Vd}$ : Je te dis non + tu n'entends pas?

La cliente commence son discours en manding en demandant à son interlocuteur si elle a la monnaie de « cinq cents francs ». Ainsi, elle utilise le français pour la nommer " ma fille », une façon, pour elle, de se familiariser avec la vendeuse et de développer une certaine parenté. La réponse négative et sans appel en wolof montre que celle-ci n'apprécie pas le fait que la cliente ait fait usage du manding pour lui demander un service. Comme nous le verrons plus tard, les Manding sont réputés être des locuteurs très attachés à leur langue et avec sa forte présence dans la communication à Ziguinchor, ils tentent généralement de l'imposer aux autres. Une attitude que rejettent certains locuteurs d'autres ethnies, qui considèrent cela comme un manque de considération. Avec la réponse très directe de la vendeuse, la cliente repose sa question en utilisant cette fois-ci le wolof. En effet, voyant la manière de s'exprimer de la vendeuse, la cliente devine clairement qu'elle n'a pas apprécié son comportement linguistique. C'est la raison pour laquelle elle se montre flexible. La réponse sous la forme interrogative de la femme diola face à son interlocutrice traduit sa réprobation, c'est la raison pour laquelle elle répond de façon très ferme " mani la deedet + xana yow doo degg? " (je te dis non + tu n'entends pas?). Cependant, face à la frustration de la vendeuse, la cliente repose sa question en y introduisant le terme populaire " patron " emprunté au français et qui traduit à ce niveau une tentative de séduction ou un signe de respect envers un interlocuteur. II s'agit, pour elle, de tenter de susciter la sympathie de la vendeuse afin d'arriver à son objectif qui est d'avoir la monnaie. Ce que nous pouvons tirer de cette conversation, c'est que la cliente, qui est dans le besoin, est obligée de suivre la vendeuse dans la langue avec laquelle elle souhaite discuter. II s'agit là d'une accommodation linguistique. Malgré toutes les tentatives de la cliente qui l'appelle "ma fille », la vendeuse se montre très ferme en refusant de répondre favorablement à la demande de la cliente.

Quand le choix du client domine

L'activité commerciale exige du commerçant qu'il ait une certaine ouverture et une certaine adaptation à toutes les situations qui se présentent surtout dans le contexte de Ziguinchor, marqué par la pluralité linguistique. Le client est considéré, en quelque sorte, comme un «roi ». Ainsi, nous avons remarqué que, dans la plupart des interactions recueillies sur le marché Saint-Maur, le choix de la langue du client domine. Plus de la moitié des discussions montre l'adaptation du commerçant à son client. Quelques interactions seront analysées afin d'illustrer ce fait.

- $1 \mathrm{Vd}$ : Jana jool+ kabanan+ cent francs

- $2 \mathrm{Cl}$ : Li+been ñaata?

Alterstice - Revue Internationale de la Recherche Interculturelle, vol. 7, $n^{\circ} 2$ 
- $3 \mathrm{Vd}$ : Cent francs !

- $4 \mathrm{Cl}$ : Waññil !+ fii la jëndee dembë

- $5 \mathrm{Vd}$ : Baxna li ma jaayul si suba motax

Traduction :

- $1 \mathrm{Vd}$ : Jeune fille viens cent francs+ cent francs

- $2 \mathrm{Cl}$ : L'unité à combien?

- $3 \mathrm{Vd}$ : Cent francs !

- $4 \mathrm{Cl}$ : Réduis ! + c'est ici que j'ai acheté hier.

- $5 \mathrm{Vd}$ : C'est bon c'est par ce que je n'ai pas vendu depuis le matin.

Cette discussion montre le marchandage entre une femme wolof, originaire de Gandiole dans la région de SaintLouis, et une jeune fille diola. Nous les avons interpelées après leur échange afin de connaitre l'identité de chacune. L'interaction commence par l'appel de la commerçante qui, voyant la cliente, essaie de l'aborder en diola en utilisant des termes simples qui ne font pas appel à des compétences linguistiques approfondies " jana jool » («jeune fille viens»). En effet, comme le commerce est une activité qui requiert l'usage de techniques séductrices, les premiers mots que les gens du Nord cherchent à maîtriser une fois au marché se réfèrent aux appels, mais aussi à la façon de nommer les clients. Une fois ces éléments maîtrisés, on s'attèle à connaître la façon de dire les sommes d'argent. C'est ainsi que, dans cet échange, l'indication du prix est en diola avec la traduction en français. Malgré les tentatives de la vendeuse de séduire la cliente en lui montrant qu'elle peut parler sa langue, celle-ci préfère répondre en wolof. Comment appréhender un tel fait ? Comment comprendre la préférence de la cliente diola de laisser sa langue pour répondre en wolof jusqu'à la fin de la discussion ? Nous pouvons justifier ce comportement de la cliente par le fait que le wolof est vu par les jeunes de Ziguinchor comme la langue d'intégration à la vie urbaine, la langue des "évolués ». Cela rejoint l'idée de Bourdieu (1982, p. 13) citée plus haut qui soutient que le langage est un instrument « d'action et de pouvoir ».

Dans un autre registre, nous avons recueilli une discussion entre une vendeuse wolof et une cliente mankagne habitant le quartier Tilène. Elle se décline comme suit :

- $1 \mathrm{Vd}$ : Klian+ si kakanje ome sa jakum

- $2 \mathrm{Cl}$ : Man dégguma joola+ man mankagne laa

- $3 \mathrm{Vd}$ : Biini waay!

- $4 \mathrm{Cl}$ : ñaata ?

- $5 \mathrm{Vd}$ : Deux cents !

- $6 \mathrm{Cl}$ : Mere bi triand la

- $7 \mathrm{Vd}$ : Yòw tey lan la ?

- $8 \mathrm{Cl}$ : Wekket cinq cents amul ?

- $9 \mathrm{Vd}$ : Amna kay +

Traduction :

- $1 \mathrm{Vd}$ : Client + les kakanje (poissons) de bonne qualité sont là

- $2 \mathrm{Cl}$ : Moi je ne comprends pas diola+ moi je suis mankagne

- $3 \mathrm{Vd}$ : Viens toi aussi !

- $4 \mathrm{Cl}$ : Combien? 
- $5 \mathrm{Vd}$ : Deux cents !

- $6 \mathrm{Cl}$ : Cette mère est une truande

- $7 \mathrm{Vd}$ : Toi aujourd'hui qu'est qui te prend ?

- $8 \mathrm{Cl}$ : II n'y a pas une monnaie de cinq cents ?

- $9 \mathrm{Vd}$ : Il y en a.

Dans sa volonté de la séduire par la langue utilisée, la vendeuse wolof a abordé sa cliente mankagne en diola, car elle ignorait son identité. Pourtant, nous avions souligné le fait que le wolof sert généralement à déceler l'identité du client. L'usage du diola peut donc être motivé par la volonté de cette vendeuse, originaire du Nord, de montrer à la cliente qu'elle a la capacité de s'exprimer dans l'idiome de l'ethnie numériquement dominante à Ziguinchor. Cependant, au-delà de ses efforts réels pour parler diola, elle le fait de façon incorrecte. En effet, le déterminant diola "si » (" les ») est la marque du pluriel, donc le verbe doit être également conjugué au pluriel et on devait avoir " sose » (" sont là ». Mais, ne maîtrisant pas le diola la vendeuse wolof utilise " ome » (" est là ») qui est la marque du singulier. Devant la commerçante qui l'aborde en diola, la cliente n'hésite pas à lui rétorquer en wolof qu'elle n'est pas de cette ethnie et qu'elle est mankagne. Ainsi, la vendeuse se plaît à continuer avec un mankagne mélangé au wolof (biini waay » (« viens toi aussi »).

Cependant, au lieu de continuer dans sa langue, comme l'a introduit la vendeuse, la cliente poursuit avec le wolof, la langue de son interlocutrice. La suite de l'interaction se poursuit dans cet idiome marqué parfois par le mélange avec le français pour l'indication du prix.

\section{Le plurilinguisme fonctionnel : des stratégies minimales pour vendre}

Nous rappelons quelques résultats des travaux de Juillard sur la vie des langues à Saint-Maur réalisés dans les années 1990. Les quatre principales langues du marché sont dans cet ordre d'usage : le wolof, le diola, le poular, le manding (1995, p. 127). Le wolof, qu'on retrouve dans le tiers des interactions, est souvent utilisé en situation d'alternance. Ce qui prouve déjà sa place importante par rapport aux autres langues utilisées sur le marché, perçues comme minoritaires. Enfin, Juillard atteste que les langues locales sont présentes dans les interactions commerciales dans tous les secteurs commerciaux, car étant utilisées dans plus de $70 \%$ des interactions recueillies (p. 155). On constate dès lors que le wolof exerçait une domination relative. Juillard avait également montré comment les acteurs avaient la capacité de passer d'une langue à l'autre en fonction des situations discursives.

En ce qui concerne notre étude, il faut noter que la diversité du plurilinguisme des acteurs commerciaux en question se présente sous deux aspects principaux. D'une part, il y a une pluralité linguistique dite « parfaite » (en référence au modèle de "bilinguisme parfait » développé par F. Laroussi) et d'autre part, celle que nous nommons " accessoire ». Le plurilinguisme " parfait » est celui d'un locuteur qui a une maîtrise approfondie des langues qui composent son répertoire, alors que le plurilinguisme " accessoire » est celui d'un locuteur qui, au-delà de sa langue de groupe, a une connaissance partielle, fonctionnelle des autres langues. C'est ce dernier type qui nous intéresse ici, car c'est plus répandu sur le marché du fait des usages prédominants des langues véhiculaires. Nous commençons nos analyses avec les déclarations de LM, un Wolof âgé de 45 ans et originaire de la région de Kaolack. Il est arrivé à Ziguinchor en 2013. La discussion se présente comme suit :

- EN : ñaata làkk ngay làkk yòw?

- LM14: Man+olof+français+soce bi damay tàkkale+kiriol bi+damay tàkkale

- EN : Noo deege làkk yòyu?

- LM15: Comme par exemple+ni mako deegee? Moy+buma amee klian +buy wax+dina maintenir sama bopp si mom+ba mënë xam xàlis bi+temer mom nuñu koy waxee+mille francs nuñu koy waxee+en même temps yokkal wala waññil nuñu koy waxee+ak jëlël+li+baxna+lòlu lepp dinaa ka jeem lijënti si ñom

Traduction :

Alterstice - Revue Internationale de la Recherche Interculturelle, vol. $7, n^{\circ} 2$ 
- EN : Combien de langues parles-tu?

- LM14: Moi+le wolof+le français+pour le manding je me débrouille+le créole aussi+je me débrouille

- EN : Comment as-tu appris ces langues ?

- LM15: Comme par exemple+comment je les ai apprises ? C'est+si j'ai un client+s'il parle+je retiens mon attention sur lui+jusqu'à connaitre comment il nomme l'argent+comment on nomme cinq cent+comment on nomme mille francsten même temps comment on dit augmente ou bien diminue et prend+ça+c'est raisonnable+tout cela je le cherche à travers lui

LM soutient avoir des compétences dans quatre langues qui sont : le wolof, le français, le manding et le créole. Mis à part ces deux langues, la présence de " damay tàkkale ", c'est-à-dire " je me débrouille » à la suite du manding et du créole permet à $L M$ de montrer son degré de maîtrise limitée de ces deux langues. Ces connaissances minimales peuvent se justifier par le fait que LM est wolof, une ethnie dont la langue est prédominante de la ville. Donc, il ne voit pas l'opportunité d'approfondir l'apprentissage des autres langues qui sont des parlers vernaculaires.

Son plurilinguisme partiel mais fonctionnel est sans doute lié au fait que c'est une situation normale pour un locuteur wolof de recourir à sa langue maternelle. Les langues locales, quant à elles, sont principalement apprises dans des situations d'interactions avec les clients. Il suffit, pour lui, de se concentrer sur les usages linguistiques de ses interlocuteurs. Cependant, seuls les termes en relation directe avec le commerce l'intéressent. II s'agit précisément d'expressions permettant de nommer les sommes d'argents, "xàlis bi ", mais aussi les éléments servant à marchander. Ainsi, en ce qui concerne les sommes d'argent, l'accent est mis sur les montants de base comme "temer ", " cinq cent "; " mille francs ». La connaissance de la façon dont on nomme ces deux montants est capitale dans la mesure où c'est à partir d'eux que se construisent les autres ${ }^{1}$. Quant au marchandage, quelques expressions sont apprises par les commerçants à l'image de LM. Il s'agit, entre autres, de : " yokkal wala waññil », " augmente ou diminue »; "jëlël», " prends"; «li+baxna», "ça c'est raisonnable ». La connaissance de ces éléments permet donc au commerçant non seulement de comprendre ce que dit son client, mais aussi d'avoir la possibilité de lui répondre. L'échange se limite à ces expressions, le vendeur ne pouvant pas aller plus loin.

\section{Un plurilinguisme à géométrie variable selon les secteurs du marché}

À l'intérieur d'un même espace, le plurilinguisme observé n'est pas homogène. C'est d'ailleurs dans ce sens que va Bulot en admettant la multiplicité qui caractérise la ville du point de vue sociolinguistique (2004, p. 102). Au marché Saint-Maur par exemple, certains secteurs se révèlent plus multilingues que d'autres. Ce qui met en relief la répartition fonctionnelle des langues en présence. Les pratiques linguistiques dans les grandes boutiques ne sont pas identiques à celles que l'on entend dans les étals.

Les pratiques bilingues : un rapport de pouvoir inversé

Au niveau du secteur de la vente de l'habillement, on remarque un bilinguisme construit autour du wolof et du créole. Cela est dû au fait que ce secteur est dominé par les commerçants venus du Nord. En effet, les populations locales ne s'intéressent pas au commerce. Par exemple, les Diolas préfèrent travailler dans l'administration. Les rares acteurs qui vendent relèvent du secteur de la vente des légumes et autres produits locaux. Le grand

\footnotetext{
${ }^{1}$ En wolof, "temer », " cinq cent » constitue le chiffre de base à partir duquel on obtient les multiples des centaines. II suffit juste de rajouter le chiffre à multiplier par « cinq cent ». Par exemple, on dira pour mille francs «ñar temer » tout en sachant que « ñar » signifie " deux » ou encore « ñet temer » pour " mille cinq cent ». Par ailleurs, les locuteurs préfèrent souvent dire « mille francs » au lieu de « « ñar temer».
} 
commerce est occupé par les ressortissants du Baol, du Walo et du Saloum préparés à cette activité parce qu'ils passent généralement leur enfance dans les foyers religieux à apprendre le Coran et n'ont pas d'autre formation ${ }^{2}$.

Ainsi, cet usage bilingue est justifié par le fait que le wolof est non seulement la principale langue commerciale mais surtout celle des acteurs dominants. II ne s'agit pas de maitriser plusieurs langues pour mieux vendre, mais d'en connaître les principales, notamment le wolof et le créole. Les autres telles que le manding, le diola, le peul, qui sont moins utilisées, ne servent généralement qu'à indiquer des prix ou à marchander. Seuls les Créoles, qui ne connaissent que leur langue, l'imposent aux commerçants. C'est ce que déclare KH :

- KH18: Kiriol ak wolof+parce que suñu njaay bi nii+kiriol yi ñoy gënë jënd kua+ dañuy joge sen dëkk ñëw+chaque jourñom dañuy ñëw di jënd en gros di yoobu+ en plus Kiriol bi rekk ngay gis+ñom dañuy ñëw duñu dégg olof+joola yi sax ñom buñu ñëwee+dinañu mënë exprimeuen olof+mais kiriol yi buñu ñëwee+ñom dañuy and en groupe boo xamanteni duñu làkk olof+léegi ñoñu+fok nga làkk ak ñom kreol+c'est obligatoire nga degg kreol

Traduction :

- KH18 : Le créole et le wolof+parce que notre commerce-ci+ce sont les Créole qui achètent le plus quoi+ils quittent leurs villes+et chaque jour ils viennent acheter en gros et retournent chez eux+en plus ce sont seulement les Créoles qui viennent et qui ne comprennent pas wolof+contrairement aux Diola+qui peuvent s'exprimer en wolof+mais les Créoles les Créoles viennent généralement en groupe et ne comprennent pas wolof+maintenant avec ça+il faut que tu parles créole avec eux $+c^{\prime}$ est obligatoire que tu comprennes le créole.

Ainsi, KH reconnaît la domination du wolof et du créole, une situation due, selon lui, à la présence massive de clients originaires de la Guinée-Bissau qui viennent souvent "en groupe " pour acheter " en gros ». L'arrivée massive de ces populations réputées être des locuteurs généralement monolingues, mais qui achètent des produits en grande quantité, justifie donc la nécessité d'apprendre leur langue car l'enjeu commercial est de taille. Pour mieux expliquer l'exigence pour les acteurs commerciaux de savoir parler créole, notre interlocuteur fait une opposition entre les Créoles et les Diolas. En effet, contrairement à ces derniers, qui sont flexibles dans la mesure où ils peuvent "s'exprimer en wolof ", les Créoles ne parlent que leur langue. Pour faire face, les commerçants sont contraints d'apprendre leur idiome, d'où l'expression " c'est obligatoire ». Cette attitude des Créoles confirme à juste titre l'idée de Bourdieu (1982. p. 14) comme quoi "les échanges linguistiques sont aussi des rapports de pouvoir symbolique où s'actualisent les rapports de force entre les locuteurs ou leurs groupes respectifs ».

$J B$, une femme d'ethnie mankagne, déclare aussi à propos du bilinguisme wolof-créole :

- JB16 :Li fi ëp daal+moy olof+à l'heure actuelle kreol+tam bëri na fi+par ce que Guinéens yi dañi ñëw fii di jënd marchandise+ngay degg nit ñu bëri+ñi deggulon kreol+leegi dañu dor dake+ngay degg ñu lay wax+keku miski

Traduction :

- JB16 : La langue la plus parlée ici + c'est le wolof + à l'heure actuelle le créole + aussi occupe une place importante + parce que les Guinéens viennent acheter de la marchandise + et tu vois plusieurs personnes + qui ne parlaient pas créole + maintenant ils parlent un peu + tu entends ceux qui te disent + keku misti ?

Dans sa réponse, JB affirme clairement la domination du wolof à travers notamment l'indice temporel "à l'heure actuelle ». La présence du créole comme deuxième langue est aussi admise. Une situation résultant, selon elle

\footnotetext{
${ }^{2}$ Dans les marchés sénégalais, on constate que la plupart des personnes qui pratiquent l'activité commerciale sont originaires des grandes cités religieuses. En effet, depuis l'enfance, ils sont envoyés dans les écoles coraniques avec un objectif double : la maîtrise du Coran et la formation sociale de l'individu. Il y a d'ailleurs le fait qu'à Touba, la cité de la confrérie religieuse Mouride qui constitue aussi la ville la plus peuplée du pays, il n’y a toujours pas d'école française. Après avoir mémorisé le livre saint, les jeunes investissent le secteur commercial. Par contre, dans certaines villes comme Ziguinchor, c'est la situation contraire, le fort taux de scolarisation fait que les jeunes préfèrent travailler dans l'administration. Ils sont souvent dans la fonction publique. En plus, une des ethnies majoritaires de la Casamance, les Diolas, conçoivent l'activité commerciale comme étant illicite, c'est-àdire qui fait appel à la tromperie, à la duperie.
} 
également, de la présence massive de locuteurs originaires de la Guinée-Bissau, c'est à dire "Guinéens yi » qui viennent acheter en gros la "marchandise " destinée à la revente chez eux. L'expression populaire " dor dake ", qui a le sens de "se débrouiller ", illustre les efforts que font les commerçants surtout nordistes pour faire face aux exigences des clients originaires de la Guinée-Bissau. L'alternance interphrastique notée à travers la phrase interrogative créole "keku misti ? ", "ça coûte combien ? " justifie la maîtrise partielle du créole par les commerçants qui, comme nous l'avons vu, ne s'intéressent le plus souvent qu'à connaître la façon de nommer les éléments qui interviennent dans leur activité.

Les pratiques plurilingues : un jeu de langues, un jeu avec les langues

En ce qui concerne le domaine de la vente des fruits, des légumes et des autres produits locaux, la situation est toute autre. Ce secteur est majoritairement occupé par les populations locales, avec surtout celles venues des villages environnants pour la vente quotidienne des produits issus du jardinage et de l'agriculture familiale. Ici, les autochtones dominent. Dès lors, les pratiques linguistiques présentent des dissemblances. Elles révèlent un certain rapport de force entres les idiomes en présence. Le plurilinguisme devient donc un enjeu de taille au niveau de la périphérie.

L'interaction suivante permet d'illustrer la place des langues dans le secteur d'hétérogénéité linguistique comme celui de la vente des légumes et poisson. Voici l'extrait d'un échange entre une jeune femme diola et une vendeuse manjaque.

\section{- $1 \mathrm{Cl}$ : Fatu+wa u tebbi ka noomen siwol sata fuken?}

- 2 Vd : Ay? Ñe+jakum ujool taate.

- $3 \mathrm{Cl}$ : Jaayma ñeti saam ++ yòw +suba dina ñëw marché?

- 4 Vd : Yòw+ suba fete la+ suba mankagne yëp dañuy noce ak pâques bi

Traduction :

- $1 \mathrm{Cl}$ : Fatou + qu'est ce qui te fait vendre du poisson d'hier?

- 2 Vd : Qui? Maintenant+ ne viens plus ici.

- $3 \mathrm{Cl}$ : Vends moi trois tas++toi demain viendras-tu au marché?

- $4 \mathrm{Vd}$ : Toi demain c'est une fête+ demain tous les Mankagne vont faire la noce avec la Pâques.

La discussion commence par une petite taquinerie amicale, une entrée en matière à la relation commerciale et au marchandage de la cliente qui demande à la vendeuse la raison pour laquelle elle vend du poisson de la veille.

L'usage du diola dans cet échange est révélateur des intentions de la cliente. En effet, connaissant l'identité de la vendeuse et croyant qu'elle ne pourrait pas comprendre son propos en diola, la cliente utilise sa langue de groupe pour taquiner la vendeuse. À sa grande surprise, celle-ci lui répond avec un diola parfait « Ay ? ñe jakum ujol taate ", traduit par "Qui? Maintenant ne viens plus ici ». On comprend par-là que la cliente utilise le diola pour dire ce qu'elle pense et qu'elle ne souhaite pas que son interlocutrice comprenne. La suite de l'interaction est exprimée en wolof. Ainsi, après lui avoir dit le nombre de tas de poisson qu'elle souhaite acheter, la jeune femme diola lui demande si elle sera au marché le lendemain. Ici, le diola sert aux discussions amicales, alors que le wolof à celles purement commerciales.

L'usage des termes comme "fête ", " noce ", " pâques " qui sont des emprunts du wolof au français, illustre la parfaite cohabitation entre les religions à Ziguinchor. En effet, même si elle n'est pas de confession chrétienne, la vendeuse déclare qu'elle ne viendra pas au marché à cause de la fête de Pâques. Cela constitue, pour elle, une façon de montrer tout son respect à l'endroit de la communauté chrétienne. II faut rappeler également qu'à Ziguinchor les fêtes chrétiennes et musulmanes sont célébrées dans la communion, la fraternité, la solidarité et sans distinction d'appartenance religieuse. 
Un autre échange met en exergue les langues locales casamançaises à savoir le manding et le diola avec des emprunts au wolof.

- $1 \mathrm{Vd}:$ Klian kortanante+

- $2 \mathrm{Cl}$ : Sumaay klian+ kata sindëëyi ?

- 3 Vd : Saaful tanta+ kòku bo+

- $4 \mathrm{Vd}$ : U nom si wol si jakkum

- $5 \mathrm{Cl}$ : Grawul i nonnom i ban

Traduction :

- $1 \mathrm{Vd}$ : Cliente comment vas-tu ?

- $2 \mathrm{Cl}$ : ça va cliente+ et la famille?

- $3 \mathrm{Vd}$ : Bonjour Tante + ils sont là-bas

- $4 \mathrm{Vd}$ : Achète du bon poisson.

- $5 \mathrm{Cl}$ : Ce n'est pas grave j'ai déjà acheté.

Nous avons dans ce passage une discussion commerciale entre une vendeuse d'ethnie manding et une cliente diola. Ainsi, dès les premiers instants de l'interaction, la vendeuse tente d'approcher sa cliente en utilisant le manding. N'étant pas de cette ethnie, celle-ci préfère répondre à la salutation en diola, sa langue de groupe, et la suite de la discussion se poursuit dans cet idiome jusqu'à la fin. Il s'agit dans cette situation de l'adaptation de la vendeuse à la cliente, un fait qui s'inscrit dans le cadre du phénomène de l'accommodation linguistique dont la théorie « suggère que ceux qui désirent l'approbation des autres s'adaptent davantage que ceux qui n'en ont pas besoin ou ne la désirent pas » (Moreau, 1997, p. 13).

L'interprétation qu'on peut faire de cette interaction principalement effectuée en manding et en diola est que leur maîtrise par la vendeuse semble effective. Elle passe immédiatement de l'une à l'autre sans hésitation, même si les Manding sont réputés être des locuteurs très "centrés " sur leur langue, c'est-à-dire moins flexibles. Ce fait démontre, néanmoins, que les représentations que la plupart des acteurs se font des locuteurs manding ne sont pas toujours valables, car ici, la vendeuse passe de sa langue au diola. Ce qu'il faut reconnaître, c'est que la volonté de la vendeuse de vendre fait qu'elle s'adapte à la cliente qui recourt au diola. Cela fait suite à la thèse de Juillard (1995, p. 132) faisant état, même sans avoir eu à le démontrer, du fait que "l'adaptation d'un Diola vers un Manding est probablement plus fréquente que l'adaptation inverse ".

Par ailleurs, dans la phrase de la cliente "Grawul i nonnom i ban », c'est-à-dire " Ce n'est pas grave, j'ai déjà acheté ", deux langues sont alternées. Il s'agit du wolof " grawul » et du diola « i nonnom i ban ». En effet, le terme " grawul » qui vient du français " grave », est adopté par le wolof en changeant la consonne [v] en [w], car le [v] n'existe pas dans l'alphabet de cette langue sénégalaise. II s'agit d'un emploi de " graw " auquel est adjoint le suffixe " ul » traduisant la négation en wolof. Le diola emprunte intégralement ce terme au wolof. C'est la raison pour laquelle la cliente l'emploie dans la phrase.

\section{Conclusion}

Actuellement, la vie des langues à Saint-Maur où le plurilinguisme était jadis une norme (Juillard, 1995) révèle une nouvelle configuration linguistique construite autour du wolof, qui domine du point de vue de son usage dans tous les secteurs notamment dans celui de la vente des produits alimentaires occupé par les autochtones. Cela traduit ce que Calvet (1994, p. 11) annonçait déjà en affirmant que "la ville est un facteur d'unification linguistique ". L'interprétation des données a révélé que l'usage du wolof s'est amplifié grâce surtout à la domination des gens du Nord qui ont fini par monopoliser l'activité commerciale. 
Cette recherche nous a permis de comprendre les dynamiques linguistiques actuelles sur le marché Saint-Maur. Contrairement aux recherches des années 1990 qui faisaient état de la tendance plurilingue de la majorité des acteurs commerciaux s'activant sur le marché Saint-Maur, notre recherche a révélé une évolution du contexte avec notamment de nouvelles dynamiques. En effet, du plurilinguisme individuel tant chanté par Juillard, nous arrivons à un plurilinguisme ciblé, fonctionnel, qui consiste pour les acteurs à n'apprendre que les phrases et mots des langues des clients qui leur permettent de faire aboutir leur vente. Il s'agit notamment d'expressions-types intervenant dans le contact et la négociation (les salutations, les appels, les sommes d'argent, le marchandage, etc.). Une autre particularité décelée dans cette étude est relative à l'hétérogénéité des pratiques plurilingues avec principalement deux angles. D'une part, nous avons identifié un bilinguisme en action, composé du wolof, la langue principale du marché, et du créole, celle qui pendant longtemps a rivalisé avec le wolof dans la communication urbaine. On les retrouve surtout dans le secteur de la vente du prêt-à-porter, des tissus et autres produits manufacturés occupés généralement par des migrants venus du nord du Sénégal. Le retour du créole portugais sur le marché se justifie par le fait que les Guinéens constituent une clientèle privilégiée pour les commerçants de Casamance. Ils viennent par groupe pour se ravitailler en marchandises destinées à la revente sur leurs marchés. Et puis, ces locuteurs sont réputés ne parler que leur idiome à Saint-Maur. C'est la raison pour laquelle les commerçants s'efforcent d'apprendre le créole pour attirer cette clientèle prestigieuse. D'autre part, il y a le secteur de la vente des produits alimentaires où nous avons un multilinguisme très présent, car c'est un secteur occupé par les populations locales.

La conséquence de ce multilinguisme à géométrie variable est que nous assistons à l'amplification du phénomène de l'alternance des langues, stratégie à laquelle ont recours ces divers acteurs commerciaux pour s'adapter à la diversité. Il s'agit là d'une stratégie discursive de contournement et de bricolage palliant l'absence de connaissances linguistiques des interlocuteurs (vendeurs ou acheteurs) dans certains idiomes.

Juillard (1990a, p. 155) avait souligné que, malgré la percée du wolof, les langues locales, principalement le manding, le poular et le diola, étaient utilisées à $70 \%$ dans les interactions. Cet article a montré que le wolof a gagné du terrain à Ziguinchor et surtout sur le marché Saint-Maur. Pour ce qui est du créole, qui était parlé comme langue de contact entre populations originaires de la Guinée-Bissau (Juillard, 1995, p. 129), elle est devenue une langue véhiculaire dans les grandes boutiques de St-Maur. Si la montée en puissance de la langue véhiculaire, le wolof, a provoqué le recul des autres idiomes, en revanche on a pu démontrer que les autres langues y sont parlées et que les marchands dans les différents secteurs tentent de s'adapter à la pluralité linguistique de leurs interlocuteurs.

On peut conclure que la situation du plurilinguisme sur ce marché varie d'un secteur commercial à l'autre et occupe donc des niches au cœur même de la domination du wolof, pourtant langue nationale officielle. Nos analyses confirment et infirment la fois l'idée de Calvet $(2002$, p. 167) qui affirme que " la ville fonctionne comme une pompe qui aspire du plurilinguisme et recrache du monolinguisme ».

\section{Références bibliographiques}

Alvir, S. (2015). Ville côté jardin ville côté cour. Approches visuelles en sociolinguistique urbaine. Paris : L'Harmattan.

Agence nationale de la statistique et de la démographie du Sénégal (ANSD) (2013). Recensement Général de la Population et de l'Habitat, de l'Agriculture et de l'Elevage (RGPHAE). Dakar : ANSD.

Bourdieu, P. (1982). Ce que parler veut dire. L'économie des échanges linguistiques. Paris : Fayard.

Beauvois, C., Blanchet, P. et Bulot, T. (dir). (2001). Sociolinguistique urbaine. Variation linguistique : images urbaines et sociales. Rennes: Presses universitaires de Rennes.

Bulot, T. et Bauvois, C. (2004). Présentation générale. La sociolinguistique urbaine : une sociolinguistique de crise ? Premières considérations, dans Lieux de ville et identité (Perspectives en sociolinguistique urbaine) (vol. 1). Paris : L'Harmattan.

Bulot, T. et Veschambre, V. (2004). Sociolinguistique urbaine et géographie sociale : hétérogénéité des langues et des espaces. Communication au colloque Espace et société aujourd'hui, Rennes, 21-22 octobre. 
Calvet, L.-J. (1981). Les langues véhiculaires. Paris : Presses universitaires de France (QSJ n 1916).

Calvet, L.-J. (1991). Le facteur urbain dans le devenir linguistique des pays africains Le facteur linguistique dans la constitution des villes africaines. Cahier Sciences Humaines, 27 (3-4), 411-432.

Calvet, L.-J. (1995). Les voix de la ville. Introduction à la sociolinguistique urbaine. Paris : Payot/Rivages.

Calvet, L.-J. (2002). Le marché aux langues : les effets linguistiques de la mondialisation. Paris : Plon.

Calvet, L.-J. (2005). Les voix de la ville revisitées sociolinguistique urbaine ou linguistique de la ville ? Revue de I'Université de Moncton, 36(1), 9-30.

Coulon, A. (2014) L'ethnométhodologie. Paris : Presses universitaires de France.

Coulon, A. (1991). Le facteur urbain dans le devenir linguistique des pays africains Le facteur linguistique dans la constitution des villes africaines. Cahier Sciences Humaines, 27(3-4), 411-432.

Dreyfus, M. (1995). Plurilinguisme à Dakar, contribution à une sociolinguistique urbaine (Thèse de doctorat, Université René Descartes, Paris V).

Dreyfus, M. et Juillard, C. (2005). Le plurilinguisme au Sénégal. Langues et identités en devenir. Paris : Karthala.

Gomez, P. et Coly, S. (dir.). (2015). La Gambie : enjeux du plurilinguisme. Acte du colloque international organisé par la Faculté des Lettres et Sciences de l'Université de Gambie du 7 au 9 novembre 2012, tome 2. Paris : L'Harmattan.

Juillard, C. (1995). Sociolinguistique urbaine. La vie des langues à Ziguinchor (Sénégal). Paris : Éditions du CNRS.

Moreau, M.-L. (1997). Sociolinguistique. Les concepts de base. Paris : Mardaga. 